\title{
In Planta Interaction and Transreplication of Distinct Begomoviruses and their Associated Components
}

\author{
Muhammad Naeem Sattar $^{1 *}$, Zafar Iqbal ${ }^{1}$, Sherif M. El-Ganainy ${ }^{2,3}$, Ahmad A. Alamer ${ }^{2}$ and Khalid A. AlHudaib \\ ${ }^{1}$ Central Laboratories, King Faisal University, PO Box 420, Al-Ahsa 31982, Kingdom of Saudi Arabia \\ ${ }^{2}$ Department of Arid Land Agriculture, College of Agricultural \& Food Sciences, King Faisal University, Box 400, Al-Ahsa \\ 31982, Kingdom of Saudi Arabia \\ ${ }^{3}$ Vegetable Disease Department, Plant Pathology Research Institute, ARC, Giza, Egypt \\ *For correspondence: naeem.sattar1177@gmail.com; mnsattar@kfu.edu.sa
}

Received 26 February 2021; Accepted 25 March 2021; Published 10 June 2021

\begin{abstract}
The studies described here were intended to examine the transreplication and interactions abilities of a widespread ToLCNDV, and an emerging begomovirus PeLCV associated with its cognate betasatellite TbLCuB. PeLCV, a monopartite begomovirus, has been characterized from many important crops, vegetables and weeds along with its associated TbLCuB. The DNA-B of bipartite ToLCNDV genome has been successfully transreplicated by the DNA-A of different bipartite begomoviruses, albeit with low frequency. Whether PeLCV can transreplicate DNA-B of ToLCNDV is unknown. To unravel this notion, both these viruses were inoculated to the model Nicotiana benthamiana plants in all possible combinations and the in planta existence of viral components were verified by PCR and Southern blot hybridization. The results demonstrated that PeLCV transreplicated and maintained ToLCNDV DNA-B. Whereas, ToLCNDV DNA-A could not transreplicate TbLCuB. Analyses of Rep proteins structure of ToLCNDV and PeLCV revealed a structural resemblance, whereas putative iteron-binding sequences of PeLCV were compatible with the Rep-binding iterons of ToLCNDV-B. The results suggested that PeLCV and ToLCNDV DNA-B can interact synergistically and can be disastrous under field conditions. (C) 2021 Friends Science Publishers
\end{abstract}

Keywords: Begomovirus; Interaction; PeLCV; ToLCNDV; TbLCuB; Transreplication

\section{Introduction}

The viruses in the genus Begomovirus and family Geminiviridae are small geminate shaped particles containing a circular, single-stranded (css) DNA genome and depends upon a distinct whitefly species Bemisia tabaci for long-distance transmission between plants or across the fields. The majority of the New World (NW) begomoviruses genome are bipartite and cssDNA (DNA-A and DNA-B) of almost similar in size with each other (Hanley-Bowdoin et al. 2013). However, the majority of Old World (OW) begomoviruses are monopartite with a distinct DNA-A component only. The DNA-A is $~ 2800$ nucleotides (nt) in length and it characteristically encode 4 5 open reading frames (ORFs) in the two orientations, respectively. Whereas in DNA-B BC1 and BV1 are present in opposite orientation and have roles in virus movement across the cells and into the phloem, respectively (Noueiry et al. 1994). All the begomovirus-encoded genes are situated either on virion or on complementary sense and have non-coding region to separate them (called intergenic region [IR]) that includes the promoter elements. This IR is commonly conserved between both genomic components and therefore it is called common region (CR). It covers origin of replication (ori), which also have hairpin nonanucleotide (TAATATT/AC) sequences (hallmark of whole family Geminiviridae), additionally it also have iteronrelated domain where Rep binds during replication (HanleyBowdoin et al. 2000). The transreplication of associated (or occasionally non-associated) DNA-Bs or the DNA-satellites is mainly dependent on the iterons sequences in the $\mathrm{N}$ terminus of replication associated (Rep) protein of DNA-A (Hanley-Bowdoin et al. 2000). Iterons are direct repeat sequences for Rep binding, introduces a nick at the nonanucleotide sequences to start rolling circle replication (Gladfelter et al. 1997).

Some OW monopartite begomoviruses occurs in association with DNA-satellites alphasatellite and/or betasatellite. According to the current classification, alphasatellites belong to a sub-family Geminialphasatellitinae in the family Alphasatellitidae (Briddon et al. 2018). Alphasatellites carry a cssDNA genome with a size equal to half of the helper virus genome and solely encodes their own Rep protein. Sometimes, the presence of an alphasatellite necessitates the association of a betasatellite during a monopartite begomovirus infection in

To cite this paper: Sattar MN, Z Iqbal, SM El-Ganainy, AA Alamer, KA AlHudaib (2021). In planta interaction and transreplication of distinct begomoviruses and their associated components. Intl J Agric Biol 26:45-51 
the OW. However, the occurrence of monopartite begomoviruses is very limited in the NW and few studies on bipartite begomoviruses have reported the alphasatellite association (Paprotka et al. 2010; Romay et al. 2010). On the other hand, betasatellites are members of genus Betasatellite in the family Tolecusatellitidae. In the OW, betasatellites carry a smaller genome (ca $1350 \mathrm{nt}$ ) and often establish a disease complex with monopartite begomoviruses. Such associations are indispensable for their encapsidation, transmission and replication. In return, betasatellites assist their helper begomoviruses with their single ORF $(\beta C 1)$ to establish the infection, pathogenicity and to tackle plant defense responses effectively. Since their first discovery in 1999 (Saunders et al. 2000), 500 complete betasatellites sequences have been submitted in the GenBank databases, which is indicative of their importance in disease etiology. Betasatellites encode single gene product $(\beta \mathrm{C} 1)$ to commence their role to suppress both TGS and PTGS (Zhou 2013), pathogenesis (Saeed et al. 2005; Qazi et al. 2007), expanding host plants range (Amin et al. 2010), suppress host defense by down regulating certain plant hormones (Zhang et al. 2012), in planta virus movement (Saeed et al. 2007), bind to RNA/DNA (Cui et al. 2005), modulate the developmental microRNAs levels (Amin et al. 2011), form multimers and interact with hostencoded factors (Cheng et al. 2011).

The association between pedilanthus leaf curl virus (PeLCV) and tobacco leaf curl betasatellite (TbLCuB) is an emerging threat (Munir et al. 2018). PeLCV was initially identified in Pedilanthus tithymaloides, in Southeast Asia (Tahir et al. 2009). Since its first discovery, PeLCV has proliferated much and has been characterized from many important crops, vegetables and weeds (Srivastava et al. 2014; Saritha et al. 2016; Zaidi et al. 2016a; Ismail et al. 2017; Munir et al. 2018; Yasmin et al. 2017). PeLCV induces growth stunting, thick leaf veins and upward leaf curling, which become more severe in the presence of TbLCuB. On the other hands, tomato leaf curl New Delhi virus (ToLCNDV) is a common bipartite begomovirus in the territory of monopartite begomoviruses in Southeast Asia. ToLCNDV infects elite cultivars of tomato crop in Pakistan and India (Sahu et al. 2010). ToLCNDV was initially characterized about 20 years ago (Padidam et al. 1995) from solanaceous crops in India. Until now, it has been wide-spread in areas of Indonesia, Iran, Spain, Tunisia, Italy, and Morocco (reviewed by (Zaidi et al. 2016b), as a result of its cross-continent spreading. ToLCNDV and its DNA-A have the ability to transreplicate CLCuMB (Saeed et al. 2007; Iqbal et al. 2017) and TbLCuB (Shahid et al. 2021). Moreover, different virus-encoded suppressors enhanced in planta movement and titre of ToLCNDV (Iqbal et al. 2020). Moreover, betasatellite molecules are found associated with ToLCNDV (Singh et al. 2012; Jyothsna et al. 2013; Akhter et al. 2014; Hameed et al. 2017). ToLCNDV-B is required by pepper leaf curl Lahore virus to cause a symptomatic infection (Shafiq et al. 2010).
Apart from these, ToLCNDV-B has been found associated with BYVMV in okra (Venkataravanappa et al. 2015).

In natural infection, multiple viruses can be present in the same host and can interact with each other antagonistically or synergistically. This research is an investigation to empirically predict the interaction and transreplication abilities of a monopartite begomovirus, PeLCV with bipartite begomovirus, ToLCNDV and vice versa.

\section{Materials and Methods}

\section{Origins of viruses and satellites}

Infectious constructs of ToLCNDV DNA-A ([hereafter referred to as TV]; accession\# U15015), ToLCNDV DNAB ([hereafter referred to as TB]; accession\# U15016), PeLCV ([hereafter referred to as PV]; accession\# $\mathrm{AM} 712436)$ and TbLCuB ([hereafter referred to as TbB]; accession\# AM955608) (Padidam et al. 1995; Ilyas et al. 2010). All the constructs were transformed into GV3101 Agrobacterium strain using electroporation (Just et al. 2017).

\section{Agrobacterium-mediated inoculation}

Agrobacterium tumefaciens cultures bearing recombinant plasmids for each component were inoculated in $N$. benthamiana plants during 4-5 weeks along with mock inoculated controls. Five plants for each combination were inoculated on the underside of 2-3 leaves with a sterile $1 \mathrm{~mL}$ syringe using $0.5-1 \mathrm{~mL}$ inoculums as described earlier (Iqbal et al. 2017). The inoculated plants were kept in growth chambers in completely controlled environment. The plants were regularly visited to observe symptom development on a daily basis. The experiment was repeated twice.

\section{Preliminary PCR-mediated diagnostics}

After two weeks of inoculation (WI), the inoculated $N$. benthamiana plants for each combination were subjected to total genomic DNA extraction using extraction buffer (EB) available in Extract-n-Amp Plant PCR Kit (Sigma-Aldrich). Systemic leaves were partially isolated by punching the leaf between the tube and cap following the addition of $50 \mu \mathrm{L}$ $\mathrm{EB}$. The tubes were incubated for $10 \mathrm{~min}$ at $95^{\circ} \mathrm{C} /$ and $1 \mu \mathrm{L}$ template for PCR was directly taken from the supernatant in the individual PCR reactions with primers specific for each genomic component (Table 1).

\section{Genomic DNA isolation, PCR and Southern blot hybridization}

Total genomic DNA was isolated at 4WI by harvesting newly emerging leaves and a slightly changed miniprep method was used for extractions (Dellaporta et al. 1983). PCR-mediated diagnostics were performed for detection of 
each inoculated virus and/or betasatellite components using respective set of primers (Table 1). Total genomic DNA (10 $\mu \mathrm{g})$ of one plant for each combination were loaded into agarose gels (1.5\%) and electrophoresed in 1.5 X TAE buffer, respectively. Equal loading of genomic DNA was ensured for Southern blot hybridization. After electrophoresis, the DNA were transferred onto Hybond nylon membranes (Amersham, the Netherlands), probed with radioactively [P-32P] dCTP labelled PCR-amplified $\mathrm{CP}$ gene fragment for TV and PV, MP fragment for TB, and $\beta \mathrm{C} 1$ gene fragment for $\mathrm{TbB}$, respectively. The protocol from Just et al. (2017) was followed for Southern blot hybridization and phosphorimager (Bio-Rad) was used to detect hybridization signals.

\section{Results}

Healthy N. benthamiana inoculated with PV showed severe symptoms with leaf curling and stunted leaves with severity index 4 (Fig. 1A and Table 2), following (Sattar et al. 2019). The presence of PV was detected with PCR and later with Southern blot hybridization (Fig. 2A). The plants coinoculated with PV along with its associated TbB exhibited severe downward curling and crumpling of the leaves, growth stunting and vein thickening phenotype (Fig. 1B and Table 2). These symptoms progressed further and the plants showed severe stunting in growth at 4 WI with severity index 5. The infection and presence of both PV and TbB was confirmed by PCR at 2 and $4 \mathrm{WI}$ in all the inoculated plants. The presence of $\mathrm{PV}$ and $\mathrm{TbB}$ was also detected in Southern blot (Fig. 2A and C).

Co-inoculation of $\mathrm{PV}$ with $\mathrm{TB}$ in $\mathrm{Nb}$ plants led to curled leaves with thick veins comparable to TV and TB infection (Fig. 1E, Table 2). Later, at 4 WI the leaves become shorter and vein thickening and upward leaf curling phenotype was more prominent with severity index 3 . No downward leaf curling (which, in most cases, are characteristics of betasatellite presence) were observed. The presence of PV and TB was confirmed at 2 and 4 WI using PCR, respectively. Similarly, the Southern blot hybridization results also successfully detected the presence of PV and TB (Fig. 2A and D), respectively.

The plants inoculated with TVonly failed to exhibit symptoms (Fig. 1C and Table 2), however, the occurrence of TV was only confirmed with PCR but no signals were found in Southern blot hybridization (Fig. 2B). The plants inoculated with TV and TB showed leaves with thick veins and upward curling at 2 WI (Fig. 1D and Table 2). The symptoms became more prominent at 4 WI with severity index 4. The presence of both genomic components of ToLCNDV was confirmed using PCR at $2 \mathrm{WI}$ and $4 \mathrm{WI}$ in all plant inoculations. The Southern blot hybridization readily confirmed the accumulation of both TV and TB (Fig. 2B and D).

Plant inoculations with TV and TbB, failed to induce any symptoms at 2 WI. Interestingly the plants started
Table 1: Oligonucleotide primers used in the study, their target and amplicon size

\begin{tabular}{llll}
\hline Primers & Primer sequences (5'-3') & $\begin{array}{l}\text { PCR } \\
\text { product }\end{array}$ & size \\
\hline AC1048 & GGRTTDGARGCATGHGTACATG & $\begin{array}{l}\text { Coat } \\
\text { AV494 }\end{array}$ & GCCYATRTAYAGRAAGCCMAG \\
Beta01 & GGTACCACTACGCATCGCAGCAGCC & $\begin{array}{l}\text { Protein } \\
\text { Betasatellite }\end{array}$ & $\sim 1.4 \mathrm{~kb}$ \\
Beta02 & GGTACCTACCCTCCCAGGGGTACAC & & \\
PCRc1 & CTAGCTGCAGCATATTTACRARWATGCCA & T-B & $\sim 0.6 \mathrm{~kb}$ \\
PBL1v2040 & GCCTCTGCAGCARTGRTCKATCTTCATACA & & \\
\hline
\end{tabular}
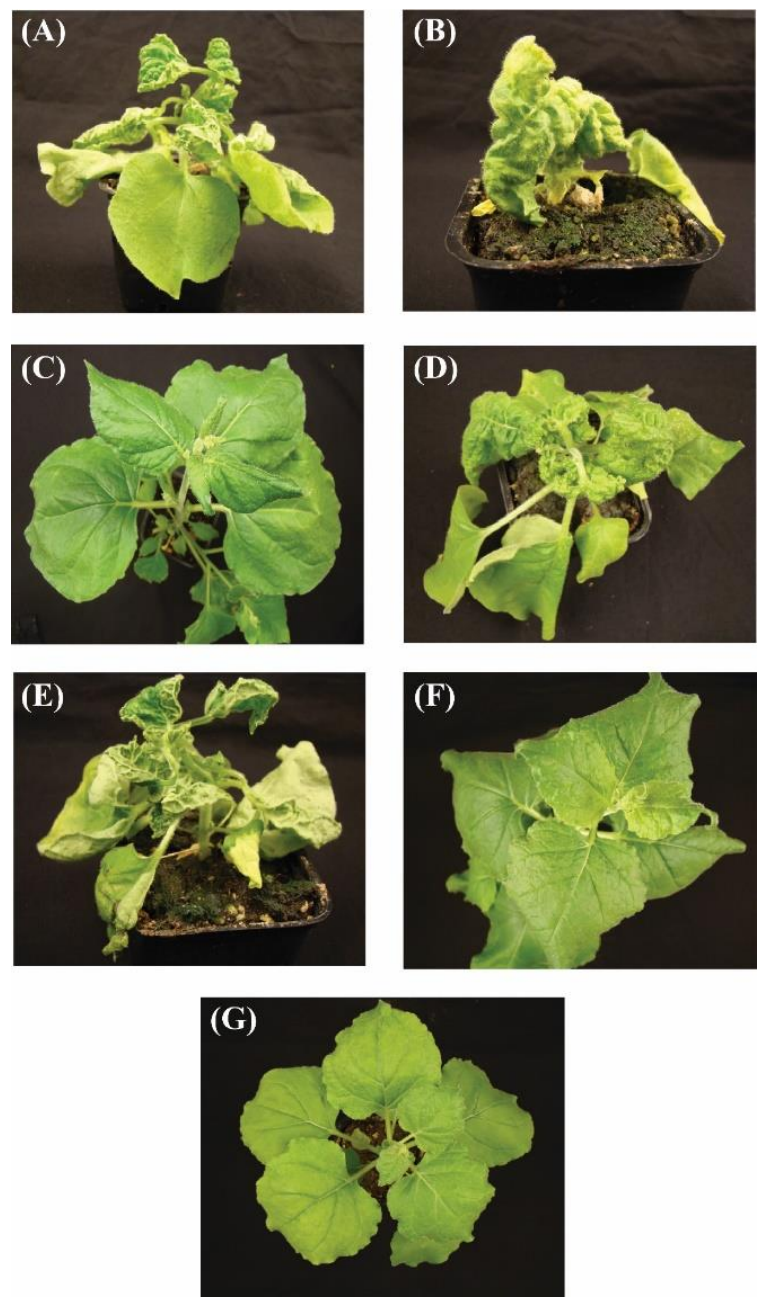

Fig. 1: Inoculation of Nicotiana benthamiana plants with PV (A), PV and TbB (B), TV (C), TV and TB (D), PV and TB (E), TV and $\mathrm{TbB}(\mathbf{F})$ and negative control $(\mathbf{G})$. All the photographs were taken at 6 weeks of post-inoculation using Sony DSC-H300 camera, after two weeks of post-inoculation. Abbreviations used are: pedilanthus leaf curl virus (PV), tobacco leaf curl betasatellite (TbB), tomato leaf curl New Delhi virus DNA-A (TV), and tomato leaf curl New Delhi virus DNA-B (TB)

showing mild leaf curling symptoms on the margins of leaves during late infection at 4 WI (Fig. 1F and Table 2). However, TV was detected successfully using PCR at 2 and 4 WI. Furthermore, the presence of TV was also detected using Southern blot hybridization (Fig. 2B); whereas PCR 
Table 2: Symptoms and severity induced by PeLCV and ToLCNDV upon inoculation with different combinations in N. benthamiana plants and Southern blot hybridization

\begin{tabular}{|c|c|c|c|c|c|}
\hline \multirow[t]{2}{*}{ Inoculation } & \multirow[t]{2}{*}{ Severity index* } & \multirow[t]{2}{*}{ Symptoms } & \multicolumn{3}{|c|}{ Southern blot hybridization } \\
\hline & & & Begomovirus & $\mathrm{TbB}$ & To-B \\
\hline PV & 4 & Severe leaf curling, growth stunting & $(+)$ & (NI) & (NI) \\
\hline $\mathrm{PV}+\mathrm{TbB}$ & 5 & Very severe leaf curling, stunted plant growth, leaf crumpling & $(+)$ & $(+)$ & (NI) \\
\hline TV & 1 & No symptoms & $(-)^{*}$ & (NI) & (NI) \\
\hline $\mathrm{TV}+\mathrm{TB}$ & 4 & Leaf curling, vein thickening, short leaves & $(+)$ & (NI) & $(+)$ \\
\hline $\mathrm{TV}+\mathrm{TbB}$ & 1 & Mild downward leaf curling & $(+)$ & ND & (NI) \\
\hline Mock control & 0 & No symptoms & ND & ND & ND \\
\hline
\end{tabular}

Pedilanthus leaf curl virus (PV), tomato leaf curl New Delhi virus (ToLCNDV), and its DNA-A (TV), and DNAB $(\mathrm{TB})$, tobacco leaf curl betasatellite $($ TbB), NI = not included, and $\mathrm{ND}=$ not detected. ${ }^{*}$ Disease severity index (0-5) was used following Sattar et al. (2019)

*Detected in PCR but not by Southern blot hybridization

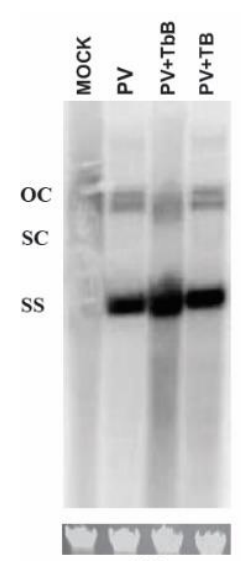

(A)

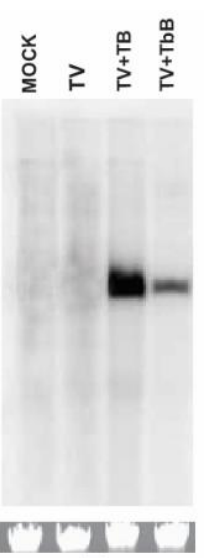

(B)

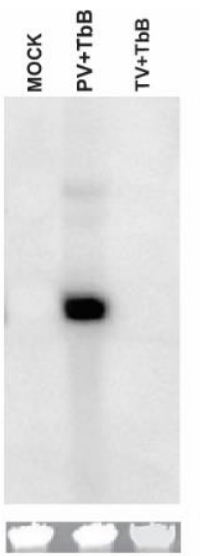

(C)

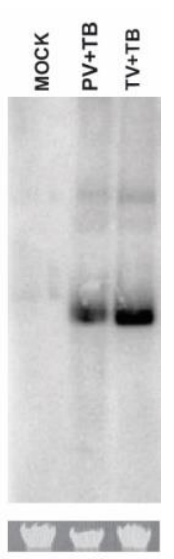

(D)

Fig. 2: Southern blot hybridization of the inoculated plants to assess trans-replication. PCR products using primers AC1048/AV494 were used as probes to detect PV (A). Lane 1: negative control, Lane 2: PV, Lane 3: PV/TbB and Lane 4: $\mathrm{PV} / \mathrm{TB}$, respectively. Similarly, $\mathrm{PCR}$ products were used as probe to detect TV (B). Lane 1: negative control, Lane 2: To -A, Lane 3: TV/TB and Lane 4: TV/TbB, respectively. The PCR products using primers Beta01/Beta02 were used to detect the presence of TbB (C). Lane 1: negative control, Lane 2: PV/TbB and Lane 3: TV/TbB. The PCR products using primer PCRc1/PBL1v2040 were used to detect the presence of TB (D). Lane 1: negative control, Lane 2: PV/TB and Lane 3: TV/TB, respectively. All the blots were probed with the $\alpha^{32} \mathrm{P}$ radiolabeled PCR products of the respective components. Different viral DNA-forms are abbreviated as open circular (OC), supercoiled (SC) and single stranded (SS) and are indicated on the right side of each panel. Other Abbreviations used are: pedilanthus leaf curl virus (PV), tobacco leaf curl betasatellite (TbB), tomato leaf curl New Delhi virus DNA-A (TV), and tomato leaf curl New Delhi virus DNA-B (TB)

and Southern blot hybridization results could not confirm $\mathrm{TbB}$ presence and replication, respectively (Fig. 2C).

Analyses of nucleotide pairwise alignment and structures of CPs encoded by TV and PV demonstrated a high sequence similarity (93.75\%) despite their less structure resemblance (Fig. 3). Whereas an opposite was observed for the Rep proteins, both Rep proteins shared less sequence similarity $(78.97 \%)$ but their structures resemblance was higher, compared to the $\mathrm{CP}$ (Fig. 3).

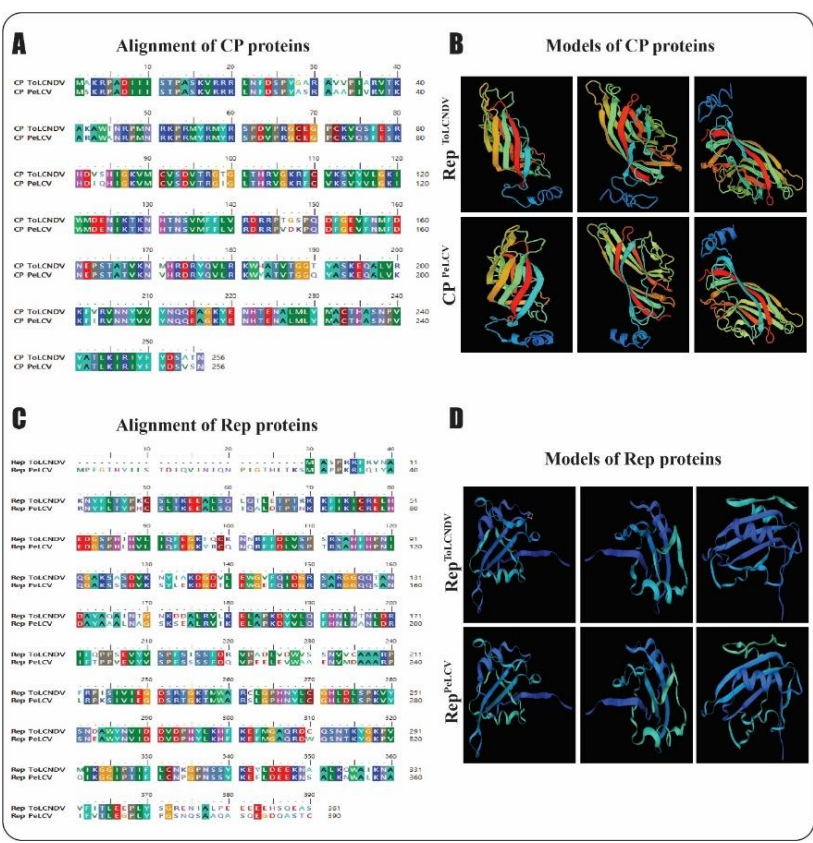

Fig. 3: Nucleotide pairwise alignment of CPs encoded by TV and PV (A) and their structure (B). Nucleotide pairwise alignment of Rep proteins encoded by TV and PV $(\mathbf{C})$ and their structure (D), respectively. Abbreviations used are: coat protein (CP), pedilanthus leaf curl virus (PV), replication associated protein (Rep), and tomato leaf curl New Delhi virus DNA-A (TV)

Analysis of the putative iteron-binding sequence (IBS) of TV, TB and PV suggested that these viral components shared highly similar and compatible Rep-binding iterons. Sequence of IBS and structural resemblance could be a contributing factor to transreplication of TB by PV.

\section{Discussion}

Mixed infections of the same or heterologous viruses in a same host plant are quite common phenomena in the fields (Annisaa et al. 2021; Ban et al. 2021). In such mixed infections, viruses interact synergistically and induce more severe symptoms than individual infection (Pruss $e t$ al. 1997), or interfere the heterologous virus infection (Crespo et al. 2020), or partially complement the infection 
of the heterologous viruses (Malyshenko et al. 1989). Additionally, during such interactions, viruses possibly recombine with each other (Hou et al. 1998; Sanz et al. 2000; Pita et al. 2001; Saunders et al. 2001) and exchange their genomic components or satellites molecules (Saunders et al. 2002; John et al. 2008). Such interactions between geminiviruses and heterologous components have been empirically proved in various studies (Sattar et al. 2019; Iqbal et al. 2020; Shafiq et al. 2021).

The results in transreplication studies showed that PV have the ability to successfully transreplicate and maintain TbB and TB. However, precise mechanism by which the putative origin of replication of TB was recognized by PVencoded Rep is unclear. The interaction between the Rep and iterons sequences in DNA-A is a determinant of the ability of helper begomovirus for DNA-B replication (Argüello-Astorga et al. 1994; Gladfelter et al. 1997). Iterons of begomoviruses vary in a way that the Rep of unrelated or same species is not allowed to perform the replication (Arguiello-Astorga et al. 1994). Two universal notions have been proposed, the "universal Rep" hypothesis states that Rep proteins may have more origin recognition relaxation properties. The second idea postulates that iteron sequences allow the Rep to recognize them; and referred to as "universal iteron" (Nawaz-ul-Rehman et al. 2009). The similarity of the IBS sequence and the structural resemblance of the Rep proteins seem to be consistent with both these hypotheses. We cannot predict the exact phenomena lying beneath, and thus it necessitates further studies. The translation of TB indicates that, regardless of an infection occurred in model plants, both PV and TV can act synergistically and potentially could break host resistance under field conditions.

Although, different betasatellites are increasingly being identified in the presence of ToLCNDV under field conditions (Bull et al. 2004; Jyothsna et al. 2013; Zaidi et al. 2016b), but TV failed to transreplicate and maintain the TbB. Although the symptomatic infection of bipartite begomoviruses require both components, rarely DNA-A is sufficient to cause asymptomatic infections on its own. (Klinkenberg and Stanley 1990; Evans and Jeske 1993; Briddon et al. 2001; Iqbal et al. 2017). The results obtained here are thus consistent to the previous findings. Possibly, this phenomenon is a peculiarity of ToLCNDV. However, further investigations are required to yield a concrete conclusion.

Nearly in all the studies investigated the interaction of a monopartite begomovirus with a cognate betasatellite resulted in plants with an enhanced titer of virus than those plants that are infected with the virus only (Saunders et al. 2000; Briddon et al. 2001; Zhou 2013). Similarly, plants coinoculated with a combination of DNA-A and betasatellite led to a more severe infection than with the plants with DNA-A infection alone (Iqbal et al. 2017). Our results confirmed these earlier reports however; our results are slightly different as replication of TV was detected in the presence of betasatellite.

In general, it seems that there is an antagonistic interaction between the betasatellite and the DNA-B. According to some previous studies, carried out using a ToLCNDV isolate and two betasatellites, a weak association between DNA-B and betasatellite was suggested, which may be due to antagonism between them (Jyothsna et al. 2013). Further studies are highly recommended to fully determine the possibility and kind of antagonistic interaction between bipartite begomoviruses and betasatellites.

\section{Conclusion}

It is likely that with intensive agriculture in southeast Asia the chances of mixed infection of multiple begmoviruses and DNA-satellites will increase. Therefore, the chances of emergence of new begomoviruses will be higher, either due to recombination (true or pseudo- recombination) or due to component/satellite capture. However, our results are corroborating with the previous studies, indicate that predicting the outcome of such encounters is a daunting task, which cannot be mapped on timescale. It will be of interest to co-inoculate different begomoviruses and DNAsatellites in different combinations to different plant species via different inoculation methods including whitefly and allow them to replicate for prolonged period to unravel the possible outcome of such interactions.

\section{Acknowledgments}

The authors extend their appreciation to the Deputyship for Research and Innovation, Ministry of Education in Saudi Arabia for the provision of funds for this research work through the project number IFT 20035.

\section{Author Contributions}

MNS, MAA and KAA designed and performed the experiment. MNS, SEE and ZI prepared the initial draft of the manuscript. ZI did pairwise sequence alignment and inferred homology models. MNS and ZI substantially improved the final copy of the draft.

\section{Conflict of Interest}

The authors declare that they have no conflict of interest.

\section{Data Availability}

All the data related to this study is included in the article, further inquiries can be directed to the corresponding author.

\section{Ethics Approval}

No humans and/or animals were used as research subject during this study. 


\section{References}

Akhter A, S Akhtar, M Saeed, S Mansoor (2014). Chili leaf curl betasatellite enhances symptoms induced by Tomato leaf curl New Delhi virus, a bipartite begomovirus. Intl J Agric Biol 16:1225-1228

Amin I, BL Patil, RW Briddon, S Mansoor, CM Fauquet (2011). Comparison of phenotypes produced in response to transient expression of genes encoded by four distinct begomoviruses in Nicotiana benthamiana and their correlation with the levels of developmental miRNAs. Virol J 8; Article 238

Amin I, M Ilyas, S Mansoor, RW Briddon, M Saeed (2010). Role of DNA satellites in geminiviral disease complexes. In: Emerging Geminiviral Diseases and their Management, pp:209-234. Sharma P, RK Gaur, M Ikegami (Eds.). Nova Science Publishers Inc. New York, USA

Annisaa NW, P Hidayat, Giyanto, SH Hidayat, S Lee (2021). Multiple infections of begomovirus on its host plants. In: IOP Conference Series on Sustainable Agriculture and Farming System: Earth Environment Science, Vol. 694, Article 012047. IOP Publishing, Bristol, UK

Argüello-Astorga GR, LR Guevara-González, LR Herrera-Estrella, RF Rivera-Bustamante (1994). Geminivirus replication origins have a group-specific organization of iterative elements: a model for replication. Virology 203:90-100

Ban F, Y Zhong, L Pan, L Mao, X Wang, Y Liu (2021). Coinfection by two begomoviruses aggravates plant symptoms but does not influence the performance and preference of insect vector Bemisia tabaci (Hemiptera: Aleyrodidae). J Econ Entomol 114:547-554

Briddon RW, DP Martin, P Roumagnac, J Navas-Castillo, E Fiallo-Olive, E Moriones, JM Lett, FM Zerbini, A Varsani (2018). Alphasatellitidae: a new family with two subfamilies for the classification of geminivirus- and nanovirus-associated alphasatellites. Arch Virol 163:2587-2600

Briddon RW, S Mansoor, ID Bedford, MS Pinner, K Saunders, J Stanley, Y Zafar, KA Malik, PG Markham (2001). Identification of DNA components required for induction of cotton leaf curl disease. Virology 285:234-243

Bull SE, WS Tsai, RW Briddon, PG Markham, J Stanley, SK Green (2004). Diversity of begomovirus DNA $\beta$ satellites of non-malvaceous plants in east and south east Asia. Arch Virol 149:1193-1200

Cheng X, X Wang, J Wu, RW Briddon, X Zhou (2011). $\beta C 1$ encoded by tomato yellow leaf curl China betasatellite forms multimeric complexes in vitro and in vivo. Virology 409:156-162

Crespo O, C Robles, L Ruiz, D Janssen (2020). Antagonism of Cucumber green mottle mosaic virus against Tomato leaf curl New Delhi virus in zucchini and cucumber. Ann Appl Biol 176:147-157

Cui X, G Li, D Wang, D Hu, X Zhou (2005). A begomovirus DNA $\beta$ encoded protein binds DNA, functions as a suppressor of RNA silencing and targets the cell nucleus. J Virol 79:10764-10775

Dellaporta SL, J Wood, JB Hicks (1983). A plant DNA minipreparation: version II. Plant Mol Biol Rep 1:19-21

Evans D, H Jeske (1993). DNA B facilitates, but is not essential for, the spread of Abutilon mosaic virus in agroinoculated Nicotiana benthamiana. Virology 194:752-757

Gladfelter HJ, PA Eagle, EPB Fontes, L Batts, L Hanley-Bowdoin (1997). Two domains of the AL1 protein mediate geminivirus origin recognition. Virology 239:186-197

Hameed A, MN Tahir, I Amin, S Mansoor (2017). First report of Tomato leaf curl New Delhi virus and a Tomato yellow leaf curl Thailand betasatellite causing severe leaf curl disease of potato in Pakistan. Plant Dis 101:1065-1069

Hanley-Bowdoin L, ER Bejarano, D Robertson, S Mansoor (2013). Geminiviruses: masters at redirecting and reprogramming plant processes. Nat Rev Microbiol 11:777-788

Hanley-Bowdoin L, SB Settlage, BM Orozco, S Nagar, D Robertson (2000). Geminviruses: models for plant DNA replication, transcription, and cell cycle regulation. Crit Rev Plant Sci 35:105-140

Hou YM, EJ Paplomatas, RL Gilbertson (1998). Host adaptation and replication properties of two bipartite geminiviruses and their pseudorecombinants. Mol Plant-Microb Interact 11:208-217
Ilyas M, J Qazi, S Mansoor, RW Briddon (2010). Genetic diversity and phylogeography of begomoviruses infecting legumes in Pakistan. $J$ Gen Virol 91:2091-2101

Iqbal Z, I Amin, S Mansoor, RW Briddon (2020). Effects of the transient expression of heterologous RNA virus-encoded silencing suppressors on the infectivity and systemic movement of tomato leaf curl New Delhi virus. Aust Plant Pathol 49:531-540

Iqbal Z, M Shafiq, I Ali, S Mansoor, RW Briddon (2017). Maintenance of cotton leaf curl Multan betasatellite by Tomato Leaf Curl New Delhi virus-analysis by mutation. Front Plant Sci 8; Article 2208

Ismail H, I Hassan, M Zubair, Z Khan, S Sarfaraz, N Jamil, S Mansoor, S Asad, I Amin (2017). First report of Pedilanthus leaf curl virus, Tobacco leaf curl betasatellite and Guar leaf curl alphasatellite infecting radish (Raphanus sativus) in Pakistan. Plant Dis 101:845

John P, P Sivalingam, Q Haq, N Kumar, A Mishra, RW Briddon, V Malathi (2008). Cowpea golden mosaic disease in Gujarat is caused by a Mungbean yellow mosaic India virus isolate with a DNA B variant. Arch Virol 153:1359-1365

Just K, MN Sattar, U Arif, A Luik, A Kvarnheden (2017). Infectivity of Tomato yellow leaf curl virus isolated from imported tomato fruit in Estonia. Zemdirbyste-Agric 104:47-52

Jyothsna P, QMI Haq, P Singh, KV Sumiya, S Praveen, R Rawat, R Briddon, VG Malathi (2013). Infection of tomato leaf curl New Delhi virus (ToLCNDV), a bipartite begomovirus with betasatellites, results in enhanced level of helper virus components and antagonistic interaction between DNA B and betasatellites. Appl Microbiol Biotechnol 97:5457-5471

Klinkenberg FA, J Stanley (1990). Encapsidation and spread of African cassava mosaic virus DNA A in the absence of DNA B when agroinoculated to Nicotiana benthamiana. J Gen Virol 71:1409-1412

Malyshenko SI, OA Kondakova, ME Taliansky, JG Atabekov (1989). Plant virus transport function: Complementation by helper viruses is nonspecific. J Gen Virol 70:2751-2757

Munir S, M Khurshid, H Kanwal, M Hussain, MN Sattar, I Ali, A Rehman, Z Iqbal (2018). Identification of pedilanthus leaf curl virus and associated betasatellite infecting turnip in Pakistan. J Plant Pathol 100:317-321

Nawaz-ul-Rehman MS, S Mansoor, RW Briddon, CM Fauquet (2009). Maintenance of an Old World betasatellite by a New World helper begomovirus and possible rapid adaptation of the betasatellite. $J$ Virol 83:9347-9355

Noueiry AO, WJ Lucas, RL Gilbertson (1994). Two proteins of a plant DNA virus coordinate nuclear and plasmodesmal transport. Cell 76:925-932

Padidam M, RN Beachy, CM Fauquet (1995). Tomato leaf curl geminivirus from India has a bipartite genome and coat protein is not essential for infectivity. J Gen Virol 76:25-35

Paprotka T, V Metzler, H Jeske (2010). The first DNA 1-like a satellites in association with New World begomoviruses in natural infections. Virology 404:148-157

Pita JS, VN Fondong, A Sangre, GW Otim-Nape, S Ogwal, CM Fauquet (2001). Recombination, pseudorecombination and synergism of geminiviruses are determinant keys to the epidemic of severe cassava mosaic disease in Uganda. J Gen Virol 82:655-665

Pruss P, X Ge, XM Shi, JC Carrington, VB Vance (1997). Plant viral synergism: the potyviral genome encodes a broad-range pathogenicity enhancer that transactivates replication of heterologous viruses. Plant Cell 9:859-868

Qazi J, I Amin, S Mansoor, J Iqbal, RW Briddon (2007). Contribution of the satellite encoded gene $\beta C 1$ to cotton leaf curl disease symptoms. Virus Res 128:135-139

Romay G, D Chirinos, F Geraud-Pouey, C Desbiez (2010). Association of an atypical alphasatellite with a bipartite New World begomovirus. Arch Virol 155:1843-1847

Saeed M, YY Zafar, JW Randles, MA Rezaian (2007). A monopartite begomovirus-associated DNA b satellite substitutes for the DNA B of a bipartite begomovirus to permit systemic infection. J Gen Virol 88:2881-2889 
Saeed M, SAA Behjatnia, S Mansoor, Y Zafar, S Hasnain, MA Rezaian (2005). A single complementary-sense transcript of a geminiviral DNA $\beta$ satellite is determinant of pathogenicity. Mol Plant-Microb Interact 18:7-14

Sahu PP, NK Rai, S Chakraborty, M Singh, PH Chandrappa, B Ramesh, D Chattopadhyay, M Prasad (2010). Tomato cultivar tolerant to Tomato leaf curl New Delhi virus infection induces virus-specific short interfering RNA accumulation and defence-associated host gene expression. Mol Plant Pathol 11:531-544

Sanz AI, A Fraile, F García-Arenal, X Zhou, DJ Robinson, S Khalid, T Butt, BD Harrison (2000). Multiple infection, recombination and genome relationships among begomovirus isolates found in cotton and other plants in Pakistan. J Gen Virol 81:1839-1849

Saritha RK, S Shrawan, P Kalia, RK Jain, VK Baranwal (2016). Association of Pedilanthus Leaf Curl Virus and satellites with carrot (Daucus carota) in India. Plant Dis 101:843-843

Sattar MN, M Ligthart, A Kvarnheden (2019). Compatibility and interaction of begomoviruses and DNA-satellites causing leaf curl disease in Asia, Africa and Mediterranean Region. Eur J Plant Pathol 155:111-124

Saunders K, N Salim, VR Mali, VG Malathi, RW Briddon, PG Markham, J Stanley (2002). Characterisation of Sri Lankan cassava mosaic virus and Indian cassava mosaic virus: evidence for acquisition of a DNA B component by a monopartite begomovirus. Virology 293:63-74

Saunders K, ID Bedford, J Stanley (2001). Pathogenicity of a natural recombinant associated with Ageratum yellow vein disease: implications for geminivirus evolution and disease aetiology. Virology 282:38-47

Saunders K, ID Bedford, RW Briddon, PG Markham, SM Wong, J Stanley (2000). A unique virus complex causes Ageratum yellow vein disease. Proc Natl Acad Sci USA 97:6890-6895

Shafiq M, MN Sattar, MS Shahid, AM Al-Sadi, RW Briddon (2021). Interaction of watermelon chlorotic stunt virus with satellites. Aust Plant Pathol 50:117-128
Shafiq M, S Asad, Y Zafar, RW Briddon, S Mansoor (2010). Pepper leaf curl Lahore virus requires the DNA B component of Tomato leaf curl New Delhi virus to cause leaf curl symptoms. Virol J 7; Article 367

Shahid MS, S Mansoor, JK Brown, RW Briddon (2021). Interaction of a tomato leaf curl New Delhi virus with a betasatellite enhances symptom severity in field-infected tomato plants. Trop Plant Pathol 46:169-174

Singh A, B Chattopadhyay, S Chakraborty (2012). Biology and interactions of two distinct monopartite begomoviruses and betasatellites associated with radish leaf curl disease in India. Virol J 9; Article 43

Srivastava A, S Kumar, SK Raj (2014). Association of Pedilanthus leaf curl virus with yellow mottling and leaf curl symptoms in two jasmine species grown in India. J Gen Plant Pathol 80:370-373

Tahir M, S Haider, J Iqbal, RW Briddon (2009). Association of a distinct begomovirus and a betasatellite with leaf curl symptoms in Pedilanthus tithymaloides. J Phytopathol 157:188-193

Venkataravanappa V, CN Lakshminarayana Reddy, S Jalali, M Krishna Reddy (2015). Association of tomato leaf curl New Delhi virus DNA-B with bhendi yellow vein mosaic virus in okra showing yellow vein mosaic disease symptoms. Acta Virol 59:125-139

Yasmin S, NI Raja, S Hameed, JK Brown (2017). First association of Pedilanthus leaf curl virus, Papaya leaf curl virus, Cotton leaf curl Kokhran virus, and Papaya leaf curl betasatellite with symptomatic chilli pepper in Pakistan. Plant Dis 101:2155-2155

Zaidi SSA, I Amin, Z Iqbal, K Pervaiz Akhtar, BE Scheffler, S Mansoor (2016a). Sesbania bispinosa, a new host of a begomovirusbetasatellite complex in Pakistan. Can J Plant Pathol 38:107-111

Zaidi SSA, M Shafiq, I Amin, BE Scheffler, JA Scheffler, RW Briddon, S Mansoor (2016b). Frequent occurrence of Tomato leaf curl New Delhi virus in cotton leaf curl disease affected cotton in Pakistan. PLoS One 11; Article e 0155520

Zhang T, JB Luan, JF Qi, CJ Huang, M Li, XP Zhou, SS Liu (2012). Begomovirus-whitefly mutualism is achieved through repression of plant defences by a virus pathogenicity factor. $\mathrm{Mol}$ Ecol 21:1294-1304

Zhou X (2013). Advances in understanding begomovirus satellites. Ann Rev Phytopathol 51:357-38 